\title{
The role of lipid geometry in designing liposomes for the solubilisation of poorly water soluble drugs
}

\author{
M. Habib Ali, Behfar Moghaddam, Daniel J. Kirby, Afzal R. Mohammed, Yvonne Perrie,
}

\section{Abstract}

Liposomes are well recognised for their ability to improve the delivery of a range of drugs. More commonly they are applied for the delivery of water-soluble drugs, but given their structural attributes, they can also be employed as solubilising agents for low solubility drugs as well as drug targeting agents. To further explore the potential of liposomes as solubilising agents, we have investigated the role of bilayer packaging in promoting drug solubilisation in liposome bilayers. The effect of alkyl chain length and symmetry was investigated to consider if using 'mismatched' phospholipids could create 'voids' within the bilayers, and enhance bilayer loading capacity. Lipid packing was investigated using Langmuir studies, which demonstrated that increasing the alkyl chain length enhanced lipid packing, with condensed monolayers forming, whilst asymmetric lipids formed less condensed monolayers. However, this more open packing did not translate into improved drug loading, with the longer chain, condensed bilayers formed from long-chain, saturated lipids offering higher drug loading capacity. These studies demonstrate that liposomes formulated from longer chain, saturated lipids offer enhanced solubilisation capacity. However the molecular size, rather than lipophilicity, of the drug to be incorporated was also a key factor dominating bilayer incorporation efficiency.

\section{Introduction}

Liposomes, due to their structural attributes, have been extensively investigated for the delivery of a wide range of drugs. Whilst more commonly used for the delivery of water soluble drugs, loading of the drug within the bilayer of the vesicles offers the potential of liposomes to act as solubilising agents. Indeed, drugs carried in the liposomal bilayer are offered the same advantages as drugs loaded within the aqueous phase of the liposomes: not only can the drug be protected from degradation in the body, the pharmacokinetic and tissue distribution patterns of the drug can also be modified, and delivery of the drug to the desired site of action can be improved. Thus liposomes are able to act not only as a stable solubilising agent, but also offer the potential for drug targeting. For example, studies from our laboratory (Daneshpour et al., 2011) have used a liposome delivery system for the site-specific delivery of novel transglutaminase (TG) inhibitors to the liver. These TG inhibitors have potential in the treatment of liver fibrosis, however they have issues with low solubility and require site specific targeting. By using anionic or neutralbased liposomes, a TG inhibitor was successfully incorporated into the bilayer of liposomes and delivered specifically to the liver (Daneshpour et al., 2011).

To continue to exploit liposomes as low solubility drug delivery systems, the underpinning parameters that control drug loading within the bilayer requires further elucidation. Liposome incorporation efficiency of low solubility drugs can be very effective, with key factors such as log $P$ and molecular size potentially influencing drug loading. Yet, liposomal incorporation of low water soluble compounds is not only dependent on the physicochemical properties of the drug; factors including bilayer composition and bilayer volume also plays a role. Indeed we have previously shown (Mohammed et al., 2004) that both the incorporation and release of a sparingly soluble drug (ibuprofen) was influenced by the composition of the liposomes, with both the choice of phosphatidylcholine and the cholesterol concentration within the bilayer having an impact on drug loading. In particular the ability of the drugs to effectively package within the liposome membrane is a dominant factor in the solubilisation of drugs within liposomes. For example, whilst cholesterol, through its ability to fill voids within a liposomal membrane, is widely accepted to improve bilayer stability (Gregoriadis and Davis, 1979), with early developmental studies (Kirby and Gregoriadis, 1980) demonstrating that an optimum of $50 \% \mathrm{~mol} / \mathrm{mol}$ cholesterol within a liposome formulation increased the stability and reduced the permeability of liposomal bilayers, the inclusion of cholesterol in liposomes used as solubilising agents requires further consideration. Indeed, studies have shown that whilst the ability of cholesterol to effectively package within a liposomal membrane can improve bilayer stability, the presence of cholesterol also counteracts the incorporation drugs within liposomal bilayers, and can reduce drug incorporation efficiency (Mohammed et al., 2004). The ability to use fatty alcohols as an alternative bilayer stabiliser to cholestero has been 
shown (Devaraj et al., 2002 and Ali et al., 2010), with the vesicles formed having similar physico-chemical characteristics, however, the fatty alcohols had a reduced impact on bilayer drug loading compared to cholesterol (Ali et al., 2010).

In addition, past studies have also suggested that the creation of 'voids' within liposomal membranes, by the appropriate selection of mis-matched lipids, can alter the physical properties of bilayers, such as fluidity and thickness. For example, Kragh-Hansen et al. (1998) discovered that when $\mathrm{C}_{12} \mathrm{NO}$ (a non-ionic surfactant) was formulated with a phospholipid, 'free volume' was created within the bilayer due to hydrophobic mismatch via the interaction between the shorter $\mathrm{C}_{12} \mathrm{NO}$ hydrocarbon chain and longer phospholipid alkyl chain. Given that the ability to incorporate a drug within a liposomal membrane is determined by appropriate molecular packing, exploiting such 'free volume' to enhance on the incorporation of hydrophobic drugs within liposomal bilayers may be advantageous. Therefore the relative significance of the structural characteristics of the membrane (i.e. alkyl chain length of lipid, geometry, nature and charge of the head-group and presence of stabiliser) must be established in order to understand the mode of action for incorporation of poorly water-soluble drugs in membranes at the molecular level (Faucon et al., 1995). It is interesting to also note that whilst nearly all liposomal formulations consider the use of symmetrical lipids, many biological lipids have asymmetric alkyl tail chains, where the tail moiety differs by their carbon number and degree of saturation, or both (Dan, 2007 and Vilcheze et al., 1996). Therefore, the aim of this work was to investigate the role of alkyl chain symmetry and geometry on the capability of liposomes as solubilisation agents. The lipids investigated are shown in Fig. 1.

\begin{tabular}{|l|l|l|}
\hline Lipid & Mol Wt & \\
\hline $\begin{array}{l}\text { 1,2-dimyristoyl-sn-glycero-3- } \\
\text { phosphocholine (DMPC) }\end{array}$ & 677 & $14: 0 / 14: 0$ \\
\hline $\begin{array}{l}\text { 1,2-dipalmitoyl-sn-glycero-3- } \\
\text { phosphocholine (DPPC) }\end{array}$ & 734 & $16: 0 / 16: 0$ \\
\hline $\begin{array}{l}\text { 1,2-distearoyl--sn-glycero-3- } \\
\text { phosphocholine (DSPC) }\end{array}$ & 734 & $14: 0 / 18: 0$ \\
\hline $\begin{array}{l}\text { 1-myristoyl-2-stearoyl-sn-glycero- } \\
\text { 3-phosphocholine (MSPC) }\end{array}$ & 760 & $16: 0 / 18: 1$
\end{tabular}

Fig. 1. Structural characteristics of lipids with symmetric and asymmetric alkyl chains lengths.

\section{Materials and methods}

Midazolam and cholesterol were purchased from Sigma-Aldrich, Dorset, UK. Propofol was supplied by MP Biomedicals, LLC, Eschwege, Germany. Egg phosphatidylcholine (PC; grade I), 1,2-dimyristoyl-sn-glycero-3phosphocholine (DMPC), 1,2-dipalmitoyl-sn-glycero-3-phosphocholine (DPPC) and 1,2-distearoyl-sn-glycero-3phosphocholine (DSPC) were purchased from Lipid Products, Epsom, Surrey, UK. Dilignoceroyl phosphatidylcholine (DGPC), 1,2-distearoyl-3-phosphatidylgycerol (DSPG), 1-palmitoyl-2-oleoyl-sn-glycero-3-phosphocholine (POPC), 1myristoyl-2-stearoyl-sn-glycero-3-phosphocholine (MSPC), 1,2-dimyristoyl-3-trimethylammonium-propane (DMTAP), 1,2-dipalmitoyl-3-trimethylammonium-propane (DPTAP) and 1,2-distearoyl-3-trimethylammonium-propane (DSTAP) were supplied by Avanti Polar Lipids, Inc., Alabaster, Alabama, USA. 


\subsection{Langmuir studies}

An automated controlled film balance apparatus (KSV Langmuir Mini-trough, KSV Instruments Ltd., Helsinki, Finland) equipped with a platinum Wilhemy plate was used to collect the surface pressure-area isotherms (Ali et al., 2010). The size of the trough was $24225.0 \mathrm{~mm}^{2}$ enclosing a total volume of about $220 \mathrm{ml}$; the sub-phase was filtered double-distilled water. The compounds (at fixed total concentration of $1 \mathrm{mg} / \mathrm{ml}$ ) were dissolved in chloroform and $20 \mu \mathrm{l}$ of each solution was spread onto the air/water interface with a Hamilton microsyringe, precise to $\pm 0.2 \mu \mathrm{l}$. After spreading, the monolayers were left for $10 \mathrm{~min}$ for the chloroform to evaporate. Thereafter, the molecules underwent constant compression until the required surface pressure was attained. Optimisation work revealed that a routine compression speed of $10 \mathrm{~mm} / \mathrm{min}$ yielded the best isotherms, therefore this speed was maintained throughout all monolayer studies. The temperature of the sub-phase was kept constant to $20 \pm 1{ }^{\circ} \mathrm{C}$ by means of an external water bath circulation system. Each experiment was only compressed once and performed at least three times with monolayers prepared from different solutions. KSV software (KSV Instruments Ltd., Helsinki, Finland) was used for data analysis.

\subsection{Preparation of multilamellar vesicles}

MLV were generated using a technique based on the established film method (Bangham et al., 1965) and modified for low solubility drugs (Mohammed et al., 2004). Briefly the lipid were dissolved in a chloroform:methanol (9:1) solvent mixture with and without the required amount of drug $(1.00 \mathrm{mg})$, and the solvent evaporated on a rotary evaporator to yield a dry film. The film was hydrated with $2 \mathrm{ml}$ of double distilled water to give final lipid concentration of $16-24 \mu \mathrm{mol} / \mathrm{ml}$ dependent on formulation.

\subsection{Determination of drug loading in liposomes}

The drug loading of liposomes was determined by measuring the non-incorporated drug present in the hydration and wash media after separation of liposomes by centrifugation (Beckman J2 Centrifuge) at $27200 \times \mathrm{g}$ for $30 \mathrm{~min}$. All samples were diluted appropriately (with respect to each drug solubility values) to avoid drug precipitation. The drug content of the supernatant was analysed by UV spectroscopy (Unicam Helios) at the appropriate wavelength for each compound (diazepam, $228 \mathrm{~nm}$; ibuprofen, $221 \mathrm{~nm}$; midazolam, $316 \mathrm{~nm}$; phenytoin, $212 \mathrm{~nm}$; propofol, $268 \mathrm{~nm}$; rifampicin, $236 \mathrm{~nm}$ ). This protocol was validated by using a direct method for extraction of drug from liposomes established by Fatouros and Antimisiaris (2002). By using this extraction method, we analysed propofol concentration within a randomly selected set of liposome preparations, whereby liposomes suspensions (after centrifugation to remove non-incorporated drug) were mixed with isopropanol in a 50/50 (v/v) mixture and solubilised by vortexing with analysis performed using UV to calculate drug concentration.

\subsection{Drug release studies}

The release rate of drug was determined by incubating drug-loaded vesicles (after separation of non-incorporated drug) in $30 \mathrm{ml} \mathrm{PBS}$ at $37^{\circ} \mathrm{C}$ in a shaking (constant) water bath. Initially, three independent samples each containing $56 \mu \mathrm{mol}$ liposomes were prepared. Each homogenous suspension was divided into seven batches, each containing 8 $\mu \mathrm{mol}$ of preparation. At time intervals of $0,2,4,8,24,48$ and $72 \mathrm{~h}$, the medium was centrifuged at $27,200 \times \mathrm{g}$ for 30 $\min$. The supernatant was analysed spectrophotometrically at the appropriate wavelength and the amount of drug released was assayed.

\subsection{Statistical analysis}

The treatment of the experimental results was based on the analysis average and the analysis of variance (ANOVA). Thereafter, Bonferroni's multiple t-test and Student-Newman-Keuls test were used to compare the difference between encapsulation efficiency, differences were considered significant when $\mathrm{P}<0.05$. Each value was obtained from at least triplicate samples and expressed as mean \pm S.D. 


\section{Results and discussion}

\subsection{Investigation of lipid packing of symmetric and asymmetric lipids using monolayer studies}

To consider the molecular packaging of the various lipids in the liposomal bilayer, monolayer studies were initially preformed. Whilst not fully representative of a bilayer, the application of monolayer studies has been effectively used to explore and identify the interactions of a range of lipids (e.g. Moghaddam et al., 2011a and Moghaddam et al., 2011b). In general, the surface pressure for the transition of a monolayer from liquid-expanded to liquidcondensed phase increased with decreasing chain length (Barnes and Gentle, 2005 and Moghaddam et al., 2011b) and this is shown in Fig. 2: for DSPC, the transition from gaseous to liquid expanded phase occurred at an approximate molecular area of $58 \AA^{2} /$ molecule while the liquid-expanded to liquid-condensed shift occurred at surface pressure of around $5 \mathrm{mN} / \mathrm{m}$, and the film collapsed at $\sim 60 \mathrm{mN} / \mathrm{m}$ (Table 1 ). The DPPC isotherm is a more expanded shape with gaseous to liquid-expanded transition taking place at $\sim 95 \AA^{2} /$ molecule (Fig. 2) and the switch from liquid-expanded to liquid-condensed occurring at a surface pressure of approximately $10 \mathrm{mN} / \mathrm{m}$, and the monolayer collapsing at about $65 \mathrm{mN} / \mathrm{m}$ (Table 1). The DMPC isotherm is the least condensed; this lipid is in a liquidexpanded phase at the start of the compression process ( $125 \AA^{2} /$ molecule) and thereon converts to the liquidcondensed phase at around a surface pressure of $18-20 \mathrm{mN} / \mathrm{m}$. The collapse pressure for DMPC is the lowest (around $55 \mathrm{mN} / \mathrm{m}$ ) compared to DSPC and DMPC. The limiting mean molecular area for DSPC, DPPC and DMPC were 51.0, 55.8 and $65.5 \AA^{2} /$ molecule, respectively (Table 1). This trend could be attributed to degree of cohesion between the chains. In this homologous series of long-chain compounds, the attraction between the chains will dictate how closely packed they arrange themselves. DSPC being the longest, will have the strongest chain-chain interactions resulting in closely packed chains (Gaines, 1966); this being reflected in its isotherm being nearly straight and steep. With decreasing chain length (i.e. DMPC $<$ DPPC $<D S P C$ ), this cohesion reduces, yielding films that become more 'gaseous' (Davies and Rideal, 1963). This work supports the rational that longer chain alkyl lipids produce more stable liposomes, since the longer alkyl chains, with stronger cohesion, results in less leakage from the aqueous core of liposomes (e.g. Mohammed et al., 2004, Taylor et al., 1990 and Moghaddam et al., 2011b).

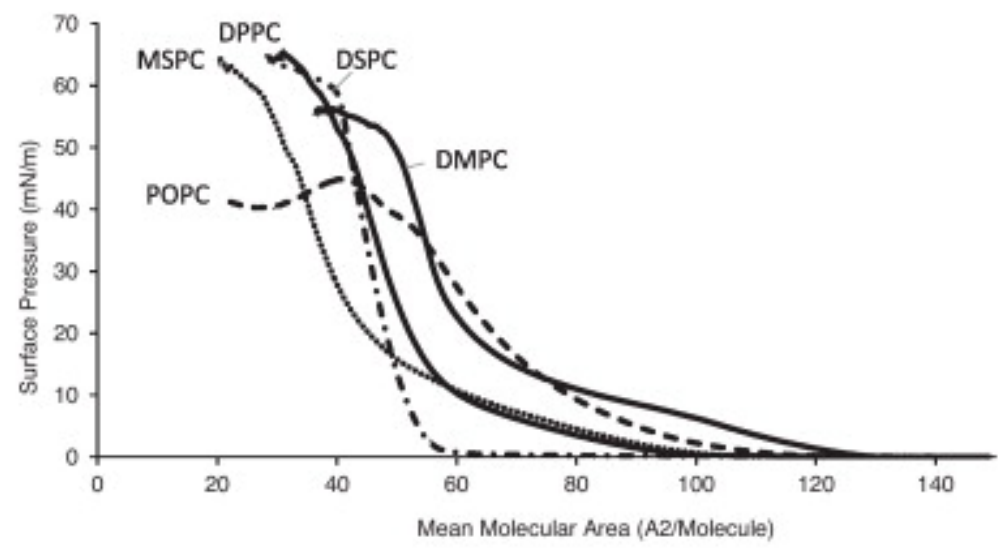

Fig. 2. The surface pressure-area isotherms of pure monolayers of DSPC, DPPC, DMPC, MSPC and POPC at the air/water interface (at $\left.20^{\circ} \mathrm{C}\right)$. Results are expressed as the means of three experiments \pm S.D.

\begin{tabular}{llll} 
Lipid category & Lipid & Extrapolated area at zero pressure $\left(\AA^{2} /\right.$ molecule $)$ & Collapse pressure $(\mathrm{mN} / \mathrm{m})$ \\
Symmetric & DSPC & 51.0 & 63.2 \\
DPPC & 55.8 & 64.3 \\
DMPC & 65.5 & 56.2 & 63.5 \\
Asymmetric & MSPC & 48.5 & \\
POPC & 80.8 & 44.9 \\
\multicolumn{2}{l}{ Table 1. Extrapolated area and collapse pressure of pure DSPC, DPPC, DMPC, MSPC and POPC monolayers at the air-water interface at } \\
temperature of $20^{\circ} \mathrm{C}$. Results are expressed as the means of three experiments.
\end{tabular}


Considering the asymmetrical lipids, the isotherm for MSPC, which has an average alkyl chain carbon number of 16 (akin to that of DPPC), bears a strong resemblance to that of DPPC (Fig. 2); gaseous to liquid-expanded transition occurred at mean molecular area $\sim 95 \AA^{2} /$ molecule with monolayer collapsing at surface pressure of about $64 \mathrm{mN} / \mathrm{m}$ (Table 1). However, the change from liquid-expanded to liquid-condensed for MSPC withstood greater surface pressures compared to DPPC and arose at approximately $18 \mathrm{mN} / \mathrm{m}$, suggesting that the presence of one 18 carbon alkyl chain was able to impact on monolayer stability. Considering the isotherm for POPC (Fig. 2), it is evident that the presence of the unsaturated bond leads to the formation of a less condensed monolayer when compared with saturated phospholipids of similar alkyl chain length. This is a result of their structure prohibiting close packaging (Gaines, 1966 and Dynarowicz-Latka and Hac-Wydro, 2004) due to the unsaturated bond in the 18-carbon alkyl chain of POPC bending the mono-unsaturated alkyl group away from the vertical 16-carbon chain. During compression, the protruding alkyl chain induces stearic hindrance, thus increasing the distance between the molecules, making close packing more difficult. In addition the number of cis double bonds in the hydrophobic alkyl chain influences its geometrical structure, with the actual number of these bonds present being proportional to how bent the chain will be ( Hac-Wydro and Wydro, 2007). In the situation of POPC, the increase in intermolecular distance reduces Van der Waal attractions between their hydrophobic regions, therefore influencing the condensation of monolayer, lowering the collapse pressure and rendering the membrane less stable ( Hac-Wydro and Wydro, 2007). This supports the suggestion that the unsaturated chain of POPC inhibits effective packaging of the lipid within the bilayer and therefore inhibits the inclusion of drugs within the bilayer, despite its longer alkyl chains lengths, and potentially increased hydrophobic volume of the liposomes in comparison to MSPC ( Fig. 2).

\subsection{Drug loading studies: selection of model drugs}

Given the wide selection of drugs which have low solubility we first pre-screened a range of commonly investigated drugs (diazepam, ibuprofen, midazolam, phenytoin, propofol and rifampicin) which were investigated in terms of their bilayer drug loading using a mulitlamellar vesicle liposome formulation previously investigated (Ali et al., 2010). These vesicles were around 5-6 $\mu \mathrm{m}$ in size, as would be expected for MLV prepared by this method (results not shown). When drug loading values were plotted against the drug lipophilicity, no apparent trend was observed (Fig. 3a), at least for the range of drugs considered here. Conversely, plotting MW against encapsulation showed some correlation (Fig. 3b), with larger molecules having lower incorporation efficiency; propofol (MW of $178 \mathrm{Da}$ ) displayed the highest bilayer incorporation of $20.3 \% \mathrm{~mol} / \mathrm{mol}$ whilst rifampicin ( $\mathrm{MW}$ of $823 \mathrm{Da}$ ) revealed the least (Fig. 3b). When considering molecules of similar lipophilicity, ibuprofen, midazolam and, propofol, with log P values of 3.48, 3.87 and 3.84, respectively (Wishart, 2006), encapsulation significantly increased in the order of propofol > ibuprofen $>$ midazolam, again reflecting their decreasing MW. Therefore it is very possible that MW plays the more influential role in dictating levels of drug encapsulation compared to drug lipophilicity, which yielded no clear correlation with respect to bilayer drug entrapment. In all the above formulations, the liposomal characteristics in terms of vesicle size and zeta potential were not significantly influenced by the drug incorporated, with the MLV being around 5-7 $\mu \mathrm{m}$ in diameter with a near neutral zeta potential (results not shown). 

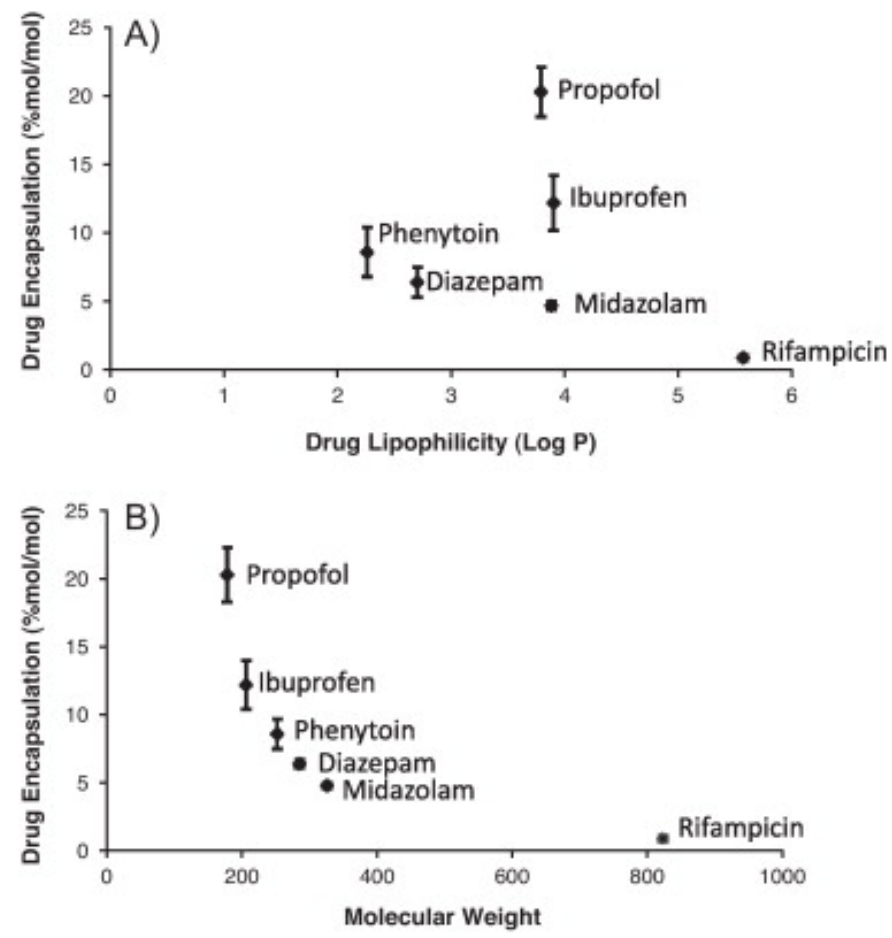

Fig. 3. The relationship between liposomal (PC:cholesterol, 89:11 mol\%) bilayer encapsulation of drug and (a) lipophilicity or (b) molecular weight of drug. Results are expressed as the means of at least four experiments \pm S.D. Results represent percentage cumulative release of initially incorporated propofol and are expressed as the means of four experiments \pm S.D.

\subsection{The effect of lipid geometry on drug solubilisation within liposomal bilayers}

To investigate the influence of bilayer area and lipid geometry on the design of liposomes as liposomal solubilising agents, liposomes prepared from a series of phospholipids, including POPC and MSPC, which are structurally asymmetrical in terms of their alkyl chain lengths, were investigated and compared with symmetrical alkyl chain lipids, DGPC, DSPC, DPPC and DMPC. Each formulation was prepared with 11 molar \% cholesterol, and the drug loading of propofol and midazolam (which offered a high and low entrapment respectively in initial studies; Fig. 3) was considered.

Considering the effect of alkyl chain length, there is a general tendency of increasing propofol and midazolam bilayer-incorporation with increased lipid chain length in the order of DGPC > DSPC > DMPC $\approx$ DPPC > PC (Fig. 4a). This correlates with previous work investigating the solubilisation of ibuprofen in liposomes (Mohammed et al., 2004) that revealed a trend of increasing encapsulation levels of water-insoluble ibuprofen with increasing lipid chain length in the order of DGPC > DSPC > DMPC > PC, with drug loading being improved by approximately $50 \%$ when DGPC was substituted for PC. In early studies, Gregoriadis (1973) showed that the longer alkyl chain DPPC was superior to PC in enhancing encapsulation of actinomycin D, a slightly water-soluble antibiotic and this trend does not appear to be exclusive to liposomes: Ismail et al. (1970) established improved solubilities of certain barbiturates in micelles of polysorbates of increasing hydrophobic chain length. This increasing drug loading, with increased alkyl chain length, has been attributed to the increased bilayer lipophilic area within systems formed by these longer lipid bilayers for the hydrophobic drug to accommodate within (Dan, 2007). 


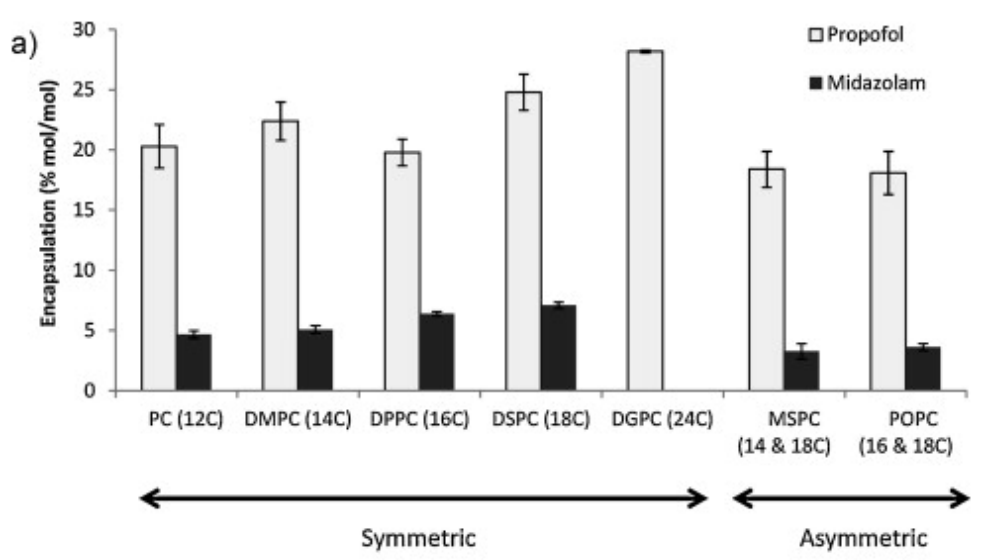

b)
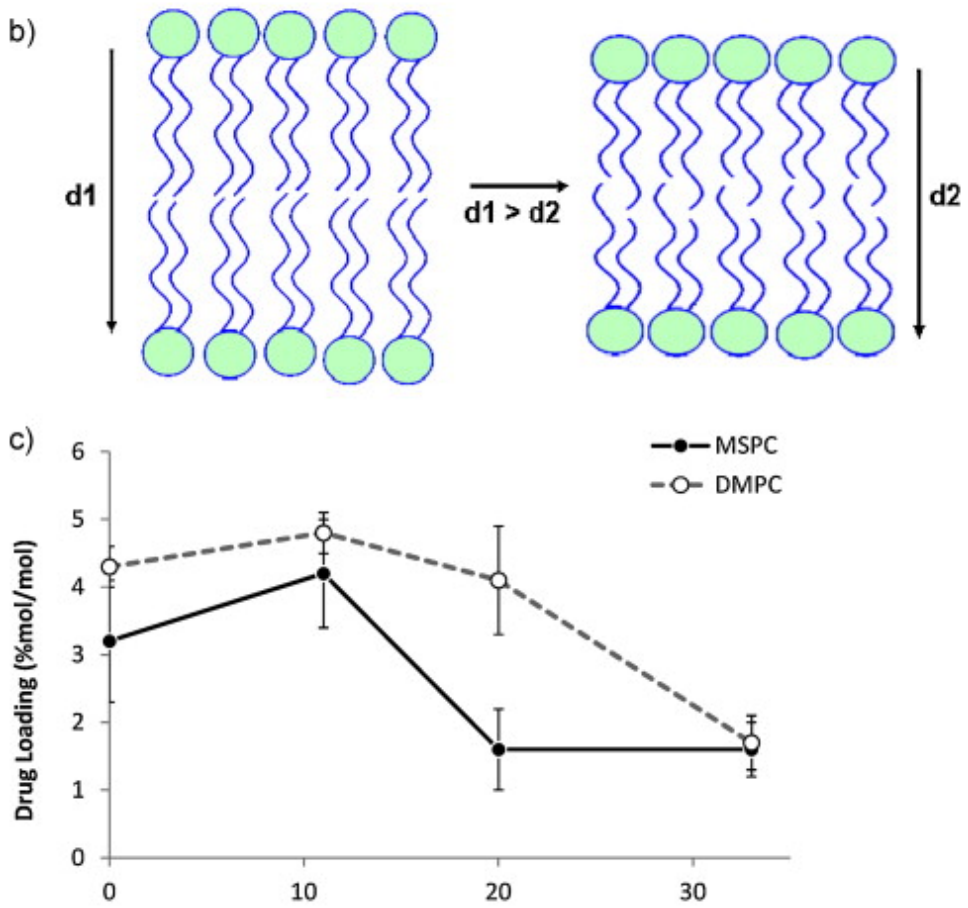

Fig. 4. The influence of long-chain symmetric and asymmetric lipids on midazolam or propofol encapsulation. (A) Liposomes were prepared from a combination of PC, DMPC, DPPC, DSPC, DGPC, POPC or MSPC (89 mol\%) with 11 total molar \% cholesterol and drug loading in a total lipid concentration of $18 \mu \mathrm{mol}$. (B) Diagram proposing the possible loss of hydrophopic region in an asymmetric lipid bilayer due to a 'mismatch' interaction between phospholipids possessing two hydrocarbon side-chains of differing length resulting in diameter reduction (right) when compared to a bilayer containing a symmetrical lipid (left) ( $d$ = diameter). (C) A comparison of midazolam encapsulation by MSPC:chol or DMPC:chol (89:11 mol\%) liposomes prepared using increasing amounts of cholesterol. Results are expressed as the means of three experiments \pm S.D.

When considering the asymmetrical PC derivatives, MSPC and POPC provided significantly $(\mathrm{p}<0.05)$ lower incorporation rates for propofol when compared to their symmetrical counterparts, DSPC and DMPC ( Fig. 4a). Similarly, incorporation studies using midazolam suggest that symmetrical PC derivatives provide significantly higher $(p<0.05)$ encapsulation of midazolam when compared their asymmetrical counterparts ( Fig. 4a). This generally reduced drug-loading by liposomes formulated from asymmetrical lipids maybe attributed to bilayer formation via a mismatch interaction between alkyl side-chains of asymmetric lipids, therefore reducing the overall hydrophobic area available for hydrophobic drug interaction, when compared to that of symmetric lipid bilayers ( Fig. 4b). The decrease in drug loading capacity of shorter alkyl chain lipid bilayers has been previously shown with incorporation of ibuprofen (Mohammed et al., 2004) and is similar to previously reported results with the micellar solubilisation of drugs such as barbiturates ( Arnarson and Elworthy, 1980 and Ismail et al., 1970). This is further supported by the finding that the presence of cholesterol within the liposome formulation has a greater impact on the MSPC formulation compared to the DMPC formulation ( Fig. 4c) suggesting the competitive space-filling action of cholesterol is more notable in the decreased bilayer volume of the MSPC liposomes. Therefore the reduced 
hydrophobic region of the MSPC combined with the competing action of cholesterol for packaging within the bilayer reduces bilayer drug loading ( Ali et al., 2010, Moghaddam et al., 2011a and Moghaddam et al., 2011b).

Given that POPC has longer alkyl chains compared to MSPC it might be expected to offer higher drug loading if the above proposal was applied, however Fig. 4a does not confirm this. The reduced drug-entrapment for POPC vesicles could be attributed to the single unsaturated bond it carries on the longer $\left(C_{18}\right)$ hydrocarbon chain. Past studies (Yoshioka et al., 1994) report that sorbitan monooleate $\left(C_{9=9}\right)$ niosomes encapsulate lower amounts of solute than its alkyl analogue, $\mathrm{C}_{18}$ sorbitan monoester niosomes. This may be due to the fact that the presence of alkyl chain unsaturation is said to enhance chain fluidity and hence bilayer permeability (Uchegbu and Florence, 1995 and Devaraj et al., 2002). Investigations using a Langmuir trough by Hac-Wydro and Wydro (2007) showed that the inclusion of a saturated fatty acid to a phospholipid/cholesterol membrane makes the model system more rigid, whereas the presence of an unsaturated fatty acid enhances its fluidity. Additionally, it is recognised that cholesterol has lower solubility in membranes with polyunsaturated acyl chain moieties (Bennati et al., 2007 and Brzustowicz et al., 2002). This can be rationalised by the fact that polyunsaturated chains display a jagged shape that does not pack adequately with the smooth surface of cholesterol (Bennati et al., 2007), thereby not rigidifying a bilayer as well as sufficiently packed cholesterol in a saturated bilayer would. Therefore, whilst the proposal that POPC liposomal bilayers may package in a way to create free space which could support bilayer drug loading (Kragh-Hansen et al., 1998), the results in Fig. 4 do not support this and it may be considered that the unsaturated chain of POPC inhibits packaging of drugs within the bilayer in a similar concept to that proposed by Bennati et al. (2007) for cholesterol.

Considering the sizes of the liposomes, encapsulating midazolam or propofol there is no notable difference between the various formulations prepared (Supplementary data; Table 1). Similarly the zeta potential was not significantly influenced by the lipid geometry as would be expected given all the lipids have the same head-group (Supplementary data; Table 1).

Given that past studies have shown that physical properties of the bilayer, such as fluidity and thickness, can be altered by creating 'voids' in the phospholipid bilayer, with Kragh-Hansen et al. (1998) demonstrating that when $\mathrm{C}_{12} \mathrm{NO}$ (a non-ionic surfactant) was formulated with a phospholipid, voids ('free volume') were formed within the bilayer due to hydrophobic mismatch via the interaction between the shorter $\mathrm{C}_{12} \mathrm{NO}$ hydrocarbon chain and longer phospholipid alkyl chain. This was further explored by combining long-chain symmetric and asymmetric lipids and DSPC was combined with POPC or MSPC, and DPPC with POPC or MSPC.

All formulations were prepared containing 11 total molar \% cholesterol (for full details on composition, see Supplementary Table). The results from Fig. 5 suggest that possible 'voids' created in the bilayer by a mismatch arrangement of phospholipids did not give a major improvement on drug solubilisation, with liposomes formulated from DSPC:cholesterol showing the highest solubilisation capacity. Similarly there was no notable difference in size or zeta potential in the various formulations (Supplementary Table 1). 


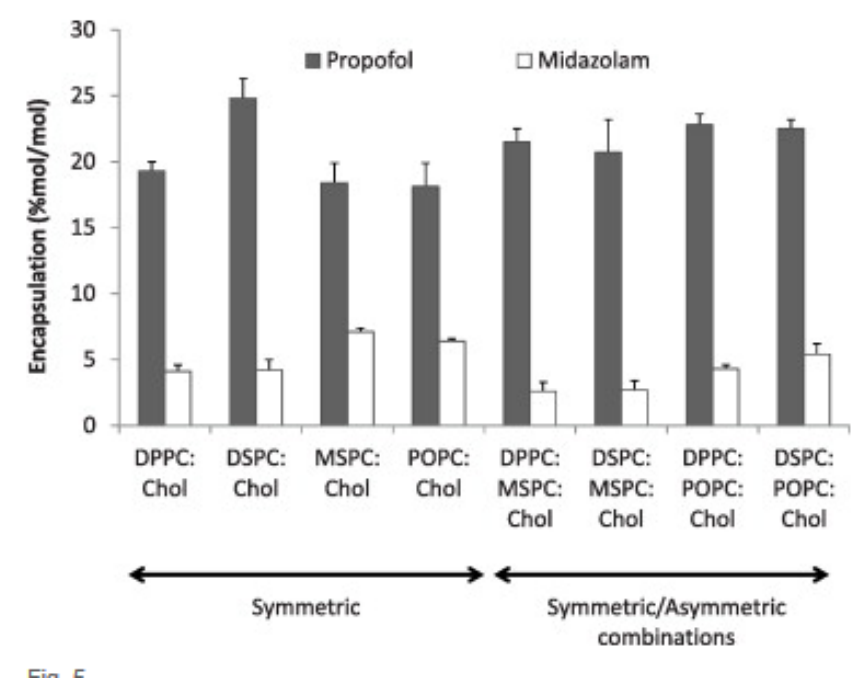

Fig. 5. The influence of combining long-chain symmetric and asymmetric lipids on drug encapsulation. Liposomes were prepared from DPPC, DSPC, MSPC or POPC and cholesterol (89:11 mol\%) or in a combination of DSPC or DPPC (44.5 mol\%) with POPC or MSPC (44.5 mol\%) and cholesterol (11 mol\%) with drug encapsulation in a total lipid concentration of $18 \mu \mathrm{mol}$ drug. Results are expressed as the means of four independent experiments \pm S.D.

\subsection{The influence of charged lipid on drug solubilisation within lipid bilayers}

Having previously shown that the presence of charged lipids influenced the solubilisation of ibuprofen, and that the presence of charged lipids may influence bilayer packing (Mohammed et al., 2004), this was further explored with propofol, using a series of cationic and anionic lipids. Liposomes were prepared from cationic lipids (DSTAP, $\mathrm{C}_{18}$ alkyl chain length; DPTAP, $C_{16}$ alkyl chain length; DMTAP, $C_{14}$ alkyl chain length) and their ability to solubilise propofol was compared against their neutral (DSPC, DPPC, and DMPC) or anionic (DSPG, DPPG, DMPG) phospholipid analogues. All formulations contained 11 molar \% cholesterol as per previous studies.

Investigations of positively charged lipids revealed a trend of increasing liposome incorporation of propofol with an increase in alkyl chain length in the order of DSTAP > DPTAP > DMTAP $(p<0.05)$ ( Fig. 6a). This trend appears to correlate with their corresponding neutral lipid derivatives ( Fig. 4a) suggesting that, irrespective of the surfactant charge, the solubilisation of propofol within the bilayer incrementally increases with increasing surfactant chain length. A similar conclusion was published by Rangel-Yagui et al. (2005) who investigated the solubilisation of ibuprofen in micellar solutions prepared from three different surfactants (sodium dodecyl sulphate, dodecyltrimethylammonium bromide and n-dodecyl octa(ethylene oxide)). 

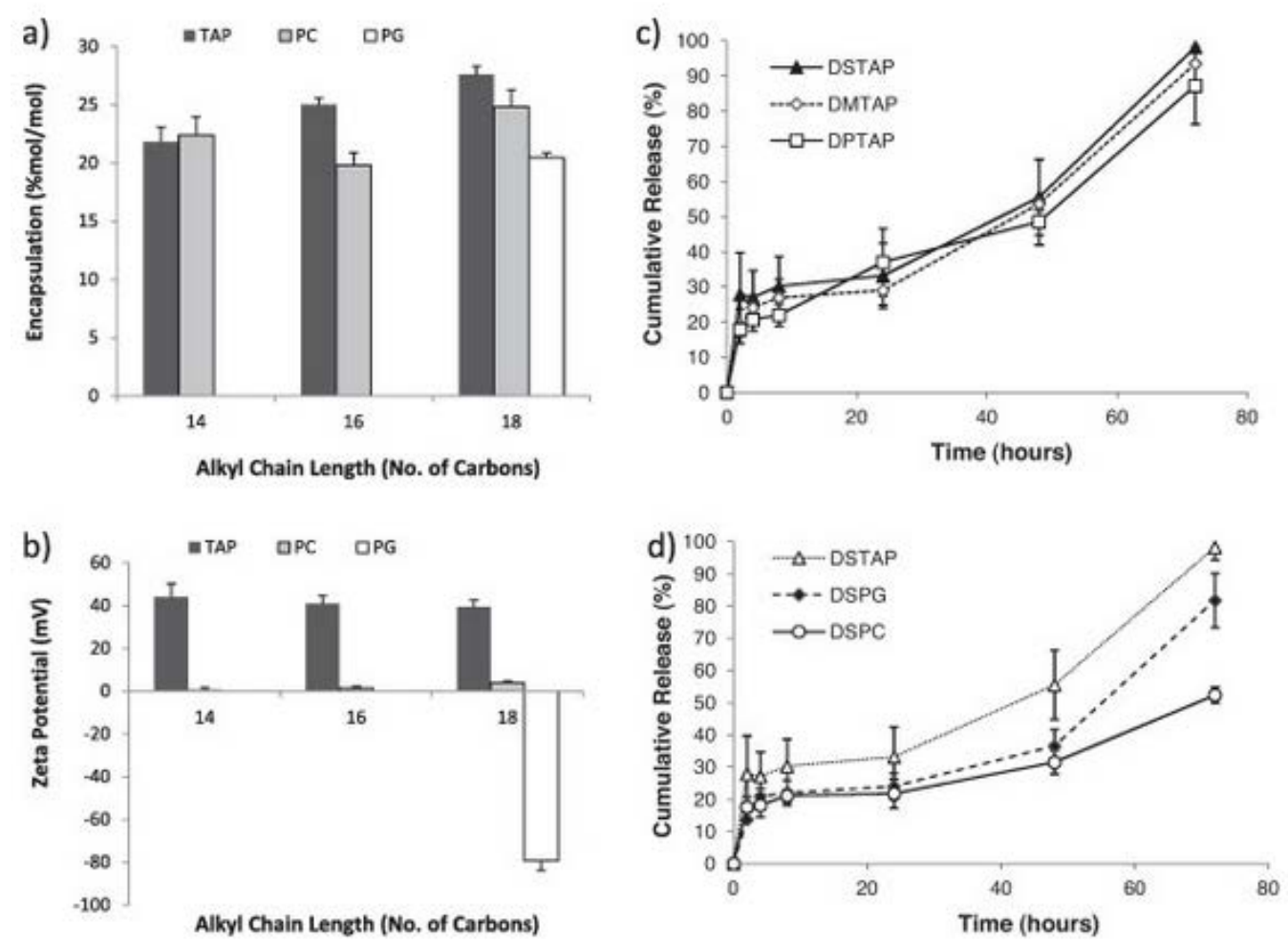

Fig. 6. (a) Encapsulation of propofol by charged liposomes and their neutral phospholipid analogue all prepared with $89 \%$ phospholipid and $11 \%$ cholesterol. (b) The vesicle surface charge of charged vesicles compared against the zeta potential of their neutral counterpart. (c) Effect of alkyl chain length of positively charged TAP series on bilayer release of propofol. (d) Effect of vesicle surface charge on rate of propofol release. Results are expressed as the means of four independent experiments \pm S.D.

The results in Fig. 6 also revealed an improvement in propofol-solubilisation by positively charged liposomes when compared with their neutral and negatively charged counterparts (Fig. $6 a$ and b). DSTAP and DPTAP encapsulated a significantly $(p<0.05)$ greater quantity of drug than their neutral analogues, DSPC and DPPC, respectively, with DMTAP revealing no significant difference in propofol-loading compared with DMPC. The increase in drugincorporation by the cationic charged liposomes could be due to intermolecular $\pi$-cation interaction between the aromatic ring of propofol and the positively charged nitrogen on the head-group of the cationic lipids, a non-bonded interaction that has attracted considerable attention ( Garau et al., 2004). Benzene is non-polar, since no single carbon atom is more negatively charged than the other carbon atoms, there is no net dipole moment. However, the distribution of electrons is uneven throughout the molecule resulting in a state termed 'quadrupole' where there exists more negative charge both above and below the hexagonal plane of carbons ( Ma and Dougherty, 1997). Empirical evidence suggests that this kind of interaction exist and is relatively strong ( Garau et al., 2004). X-ray crystal analysis by Hamada et al. (2006) reveal the molecular assembly of thiacalix[6]arene via $\pi$-cation interaction between the aromatic ring of thiacalix[6]arene and a positively charged group, such as nitrogen of dimethylformamide. Furthermore, Dougherty and Stauffer (1990) proposed that the $\pi$-cation interaction is responsible for the binding of acetylcholine to its deactivating enzyme, acetylcholinesterase.

\subsection{Solubilised drug release from the liposomal carriers}

To consider how the formulation of the vesicles influenced drug release rates, three formulations, consisting of DMTAP, DPTAP or DSTAP and including $11 \mathrm{~mol} \%$ cholesterol, were incubated in PBS at $37^{\circ} \mathrm{C}$ for up to $72 \mathrm{~h}$ and their release of retained propofol examined. Results (Fig. $6 \mathrm{c}$ ) suggest that alkyl chain length of cationic lipid series (DMTAP, DPTAP and DSTAP) does not appear to influence the cumulative release of propofol. This is in contrast to the outcome observed for neutral lipid formulations previously reported (Ali et al., 2010). Therefore, the impact of the charge-headgroups was also considered. Fig. $6 \mathrm{~d}$ demonstrates that the cumulative drug released at $72 \mathrm{~h}$ varied between the DSPC, DSTAP and DSPG preparations. Of the three formulations, drug release from cationic DSTAP 
formulation was the highest and the slowest release rate was noted for the neutral DSPC preparation, suggesting that both charge and alkyl chain length are contributing to the release rates. The effect of charge on drug permeability has been previously documented; results collated by Nounou et al. (2006) revealed that positively charged liposomes exhibited the fastest release compared with neutral and negatively charged preparations. Furthermore, Mohammed et al. (2004) demonstrated that the inclusion of $9 \mathrm{~mol} \%$ stearylamine (positively charged) or dicetylphosphate (negatively charged) induced significantly more release of ibuprofen compared to neutral liposomes. Given the above findings, it is clear that electrostatic drug-bilayer interactions, in combination with lipid packaging, needs consideration in bilayer drug release.

\section{Conclusion}

Liposomes as solubilising agents can play a key role in addressing the need for improved delivery of low solubility drugs. Whilst already clinically exploited for the solubilisation and delivery of the Amphotericin B, additional understanding of the parameters which dictate incorporation of the drugs within bilayers is required. Given that the ability for drugs to package within these bilayers is a key attribute, we have investigated if the development of bilayers from 'mis-matched' lipids to create 'free space' within the bilayers can enhance drug loading. Langmuir studies revealed that both the length of alkyl chain and the presence of unsaturated bonds influenced lipid packaging in a monolayer, with shorter chain lipids and lipids with asymmetric lipid chains showing less condensed monolayers, suggesting these lipids may offer more space for solubilisation for drugs within liposomal bilayers. However, of the liposomal formulations studied, formulations containing longer alkyl chain length lipids (DSPC, DSPG and DSTAP) gave higher drug solubilisation and the use of lipids with asymmetric alkyl chains did not enhance solubilisation efficiency. This confirms that the proposal of creating voids within the liposomal bilayer is not advantageous in designing liposomes as solubilisation agents despite drug molecular size being a key factor in drug loading capacity of liposomes.

\section{Acknowledgements}

M. Habib Ali was funded the Engineering and Physical Sciences Research Council (Grant GR/S61287/01). Behfar Moghaddam was funded by an Aston University International Student Scholarship.

\section{References}

M.H. Ali, D.J. Kirby, A.R. Mohammed, Y. Perrie

Solubilisation of drugs within liposomal bilayers: alternatives to cholesterol as a membrane stabilising agentJ. Pharm. Pharmacol., 62 (2010), pp. 1646-1655

T. Arnarson, P.H. Elworthy

Effect of structural variations of non-ionic surfactants on micellar properties and solubilisation: surfactants based on erucyl and behenyl (C22) alcohols

J. Pharm. Pharmacol., 32 (1980), pp. 381-386

A.D. Bangham, M.M. Standish, J.C. Watkins

Diffusion of univalent ions across the lamellae of swollen phospholipids

J. Mol. Biol., 13 (1965), pp. 325-328

G.T. Barnes, I.R. Gentle

Interfacial Science: An Introduction

Oxford University Press Inc., UK (2005)

C.R. Bennati, R.M. Epand, M.T. Lamy

Low cholesterol solubility in DODAB liposomes

Chem. Phys. Lipids, 145 (2007), pp. 27-36

M.R. Brzustowicz, V. Cherezo, M. Zerouga, M. Caffrey, W. Stillwell, S.R. Wassall Controlling membrane cholesterol content. A role for polyunsaturated (docosahexaenoate) phospholipids Biochemistry, 41 (2002), pp. 12509-12519 
Dan

Lipid tail chain asymmetry and the strength of membrane-induced interactions between membrane proteins

Biochim. Biophys. Acta, 1768 (2007), pp. 2393-2399

N. Daneshpour, M. Griffin, R. Collighan, Y. Perrie

Targeted delivery of a novel group of site-directed transglutaminase inhibitors to the liver using liposomes: a new approach for the potential treatment of liver fibrosis

J. Drug Target., 8 (2011), pp. 624-631

J.T. Davies, E.K. Rideal

Interfacial Phenomena

Academic Press Inc., New York, USA (1963)

G.N. Devaraj, S.R. Parakh, R. Devraj, S.S. Apte, B. Ramesg Rao, D. Rambhau

Release studies on niosomes containing fatty alcohols as bilayer stabilisers instead of cholesterol

J. Colloid Interface Sci., 251 (2002), pp. 360-365

D.A. Dougherty, D.A. Stauffer

Acetylcholine binding by a synthetic receptor: implications for biological recognition

Science, 250 (1990), pp. 1558-1560

P. Dynarowicz-Latka, K. Hac-Wydro

Interactions between phosphatidylcholines and cholesterol in monolayers at the air/water interface

Colloid Surf. B Biointerf., 37 (2004), pp. 21-25

D.G. Fatouros, S.G. Antimisiaris

Effect of amphiphilic drugs on the stability and zeta-potential of their liposome formulations: a study with prednisolone, diazepam, and griseofulvin

J. Colloid Interface Sci., 251 (2002), pp. 271-277

J.-F. Faucon, J.-M. Bonmatin, J. Dufourcq, E.J. Dufourc

Acyl chain length dependence in the stability of melittin-phosphatidylcholine complexes. A light scattering and 31P NMR study Biochim. Biophys. Acta, 1234 (1995), pp. 235-243

\section{G.L. Gaines}

Insoluble Monolayers at Liquid-Gas Interfaces

John Wiley \& Sons Inc., New York (1966)

C. Garau, A. Frontera, D. Quinonero, P. Ballester, A. Costa, P.M. Deya

Cation- $\pi$ vs. anion- $\pi$ interactions: a complete $\pi$-orbital analysis

Chem. Phys. Lett., 399 (2004), pp. 220-225

G. Gregoriadis

Drug entrapment in liposomes

FEBS Lett., 36 (1973), pp. 292-296

G. Gregoriadis, C. Davis

Stability of liposomes in vivo and in vitro is promoted by their cholesterol content in the presence of blood cells

Biochim. Biophys. Res. Commun., 90 (1979), pp. 1287-1293

K. Hac-Wydro, P. Wydro

The influence of fatty acids on model cholesterol/phospholipid membranes

Chem. Phys. Lipids, 150 (2007), pp. 66-81

F. Hamada, Y. Higuchi, Y. Kondo, C. Kabuto, N. Iki

Supramolecular assembly based on $\pi-\pi$ stacking $\pi$-cation interactions between thiacalixarene and DMF

Tetrahedron Lett., 47 (2006), pp. 5591-5593

A.A. Ismail, M.W. Gouda, M.M. Motawi

Micellar solubilisation of barbiturates I: solubilities of certain barbiturates in polysorbates of varying hydrophobic chain length

J. Pharm. Sci., 59 (1970), pp. 220-224 
C. Kirby, G. Gregoriadis

Effect of the cholesterol content of small unilamellar liposomes on their stability in vivo and in vitro

Biochem. J., 186 (1980), pp. 591-598

U. Kragh-Hansen, M. le Maire, J.V. Moller

The mechanism of detergent solubilization of liposomes and protein-containing membranes

Biophys. J., 75 (1998), pp. 2932-2946

J.C. Ma, D.A. Dougherty

The cation- $\pi$ interaction

Chem. Rev., 97 (1997), pp. 1303-1324

B. Moghaddam, S.E. McNeil, Q. Zheng, A.R. Mohammed, Y. Perrie

Exploring the correlation between lipid packaging in Lipoplexes and their transfection efficiency

Pharmaceutics, 3 (2011), pp. 848-864

B. Moghaddam, M.H. Ali, J. Wilkhu, D.J. Kirby, A.R. Mohammed, Q. Zheng, Y. Perrie

The application of monolayer studies in the understanding of liposomal formulations

Int. J. Pharm., 417 (2011), pp. 235-244

A.R. Mohammed, N. Weston, A.G.A. Coombes, M. Fitzgerald, Y. Perrie

Liposome formulation of poorly water soluble drugs: optimisation of drug loading and ESEM analysis of stability

Int. J. Pharm., 285 (2004), pp. 23-34

M. Nounou, L.K. El-Khordagui, N.A. Khalafallah, S.A. Khalil

In vitro release of hydrophilic and hydrophobic duugs from liposomal dispersions and gels

Acta Pharm., 56 (2006), pp. 311-324

C.O. Rangel-Yagui, A. Pessoa Jr., L.C. Tavares

Micellar solubilization of drugs

J. Pharm. Pharm. Sci., 8 (2005), pp. 147-163

K.M.G. Taylor, G. Taylor, I.W. Kellaway, J. Stevens

Drug entrapment and release from multilamellar and reverse-phase evaporation liposomes

Int. J. Pharm., 58 (1990), pp. 49-55

I.F. Uchegbu, A.T. Florence

Non-ionic surfactant vesicles (niosomes): physical and pharmaceutical chemistry

Adv. Colloid Interface Sci., 58 (1995), pp. 1-55

C. Vilcheze, T.P.W. McMullen, R.N. McElhaney, R. Bittman

The effect of side-chain analogues of cholesterol on the thermotropic phase behaviour of 1-stearoyl-2-oleoylphosphatidylcholine bilayers: a differential scanning calorimetric study

Biochim. Biophys. Acta, 1279 (1996), pp. 235-242

D.S. Wishart, C. Knox, A.C. Guo, S. Shrivastava, M. Hassanali, P. Stothard, Z. Chang, J. Woolsey

DrugBank: a comprehensive resource for in silico drug discovery and exploration

Nucl. Acids Res., 1 (January 2006), pp. D668-D672 (Database issue)

T. Yoshioka, B. Sternberg, A.T. Florence

Preparation and properties of vesicles (niosomes) of sorbitan monoesters (Span 20, 40, 60 and 80) and a sorbitan triester (Span 85)

Int. J. Pharm., 105 (1994), pp. 1-6 